Robert B. Den, Edouard J. Trabulsi, Adam P. Dicker, Costas D. Lallas, and Leonard G. Gomella, Sidney Kimmel Medical College at Thomas Jefferson University, Philadelphia, PA; Firas Abdollah, Vattikuti Urology Institute, Henry Ford Hospital, Detroit; Felix Y. Feng, University of Michigan, Ann Arbor, MI; R. Jeffrey Karnes, Mayo Clinic, Rochester, MN; and Kasra Yousefi, Voleak Choeurng, and Elai Davicioni, GenomeDx Biosciences, Vancouver, British Columbia, Canada. Published online ahead of print at www.jco.org on February 9, 2015

Terms in blue are defined in the glossary, found at the end of this article and online at www.jco.org.

Authors' disclosures of potential conflicts of interest are found in the article online at www.jco.org. Author contributions are found at the end of this article.

Both R.B.D. and K.Y. made equal contributions to this article.

Corresponding author: Robert B. Den, MD, Sidney Kimmel Medical College at Thomas Jefferson University, 111 South 11th St, Room G-301, Bodine Center, Philadelphia, PA 19107; e-mail: Robert.Den@jefferson.edu.

(c) 2015 by American Society of Clinical Oncology

0732-183X/15/3308w-944w/\$20.00

DOI: $10.1200 / \mathrm{JCO} .2014 .59 .0026$

\title{
Genomic Classifier Identifies Men With Adverse Pathology After Radical Prostatectomy Who Benefit From Adjuvant Radiation Therapy
}

Robert B. Den, Kasra Yousefi, Edouard J. Trabulsi, Firas Abdollah, Voleak Choeurng, Felix Y. Feng, Adam P. Dicker, Costas D. Lallas, Leonard G. Gomella, Elai Davicioni, and R. Jeffrey Karnes

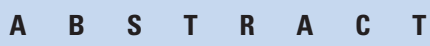

\section{Purpose}

The optimal timing of postoperative radiotherapy (RT) after radical prostatectomy (RP) is unclear. We hypothesized that a genomic classifier (GC) would provide prognostic and predictive insight into the development of clinical metastases in men receiving post-RP RT and inform decision making.

\section{Patients and Methods}

GC scores were calculated from 188 patients with pT3 or margin-positive prostate cancer, who received post-RP RT at Thomas Jefferson University and Mayo Clinic between 1990 and 2009. The primary end point was clinical metastasis. Prognostic accuracy of the models was tested using the concordance index for censored data and decision curve analysis. Cox regression analysis tested the relationship between GC and metastasis.

\section{Results}

The cumulative incidence of metastasis at 5 years after RT was 0\%, 9\%, and 29\% for low, average, and high GC scores, respectively $(P=.002)$. In multivariable analysis, GC and pre-RP prostate-specific antigen were independent predictors of metastasis (both $P<.01$ ). Within the low GC score $(<0.4)$, there were no differences in the cumulative incidence of metastasis comparing patients who received adjuvant or salvage RT $(P=$.79). However, for patients with higher GC scores $(\geq 0.4)$, cumulative incidence of metastasis at 5 years was $6 \%$ for patients treated with adjuvant RT compared with $23 \%$ for patients treated with salvage RT $(P<.01)$.

\section{Conclusion}

In patients treated with post-RP RT, GC is prognostic for the development of clinical metastasis beyond routine clinical and pathologic features. Although preliminary, patients with low GC scores are best treated with salvage RT, whereas those with high GC scores benefit from adjuvant therapy. These findings provide the first rational selection of timing for post-RP RT.

\section{J Clin Oncol 33:944-951. (C) 2015 by American Society of Clinical Oncology}

\section{INTRODUCTION}

Despite the significant stage migration in prostate cancer $(\mathrm{PCa})$ after the introduction of prostatespecific antigen (PSA) in clinical practice, a significant proportion of contemporary patients harbor adverse pathologic characteristics at radical prostatectomy (RP). ${ }^{1}$ These individuals are frequently treated with postoperative radiotherapy (RT) alone or RT plus hormonal therapy. ${ }^{2}$ However, the optimal timing of postprostatectomy RT is a subject of continuous debate. Advocates for adjuvant RT (ART) argue that this treatment modality might maximize cancer control outcomes. However, salvage RT (SRT) can minimize overtreatment while offering acceptable oncologic outcomes.
Multiple retrospective analyses have compared ART with SRT, with some studies demonstrating improvement in biochemical no evidence of disease (bNED) $)^{3,4}$ favoring ART and others indicating that early SRT (triggered at a PSA between 0.3 and 0.5 $\mathrm{ng} / \mathrm{mL}$ ) does not compromise outcomes. ${ }^{5}$ Given the even balance of the published literature, prospective randomized trials (Radiotherapy and Androgen Deprivation in Combination After Local Surgery [RADICALS], ${ }^{6}$ French Genitourinary Tumor Group trial 17/0702, ${ }^{7}$ and Radiotherapy-Adjuvant Versus Early Salvage $[\text { RAVES }]^{8}$ ) are under way comparing ART with SRT.

Because of the rarity of data in this field and the unresolved controversy between ART and SRT, we sought to integrate a novel biomarker test to 
improve clinical decision making regarding post-RP RT. We hypothesized that the use of a validated PCa genomic classifier (GC) could distinguish between men who would benefit from ART and those in whom SRT would be the optimal approach.

\section{PATIENTS AND METHODS}

\section{Patient Cohort}

The GenomeDx PCa genomic database was used to extract the data of all patients with pT3 disease and/or positive surgical margins who received post-RP RT between 1990 and 2009. A total of 198 patients had available GC scores and clinical data for nomogram computation. Ten patients (5\%) who received neoadjuvant hormonal therapy $(\mathrm{n}=3)$ and/or had lymph node invasion $(n=6)$ or who received RT after metastatic disease onset $(n=1)$ were excluded. A total of 188 patients from Thomas Jefferson University (Philadelphia, PA; $n=137$ ) and Mayo Clinic (Rochester, $M N ; n=51$ ) formed our analytic data set. ${ }^{9,10}$ Patients were treated to a median dose of 66.6 Gy using conventional fractionation by either three-dimensional conformal RT or intensity-modulated RT techniques. Photons of 10 to $25 \mathrm{MV}$ were used, with the clinical target volume delineated on computed tomography to include the prostatic fossa and periprostatic tissues. There was no statistical difference in use of intensity-modulated RT or pelvic fields or use of androgen-deprivation therapy in conjunction with RT between SRT and ART. ${ }^{11,12}$

The primary end point for the analysis was clinical metastasis (regional or distant) documented radiographically on computed tomography or bone scan. ART and SRT were defined by PSA levels of $\leq 0.2$ and $>0.2 \mathrm{ng} / \mathrm{mL}$ before initiation of RT, ${ }^{9}$ consistent with randomized clinical trials. ${ }^{6,8}$ This study follows the REMARK criteria for evaluation of prognostic biomarkers. ${ }^{13}$ The Thomas Jefferson University and Mayo Clinic institutional review boards reviewed and approved the research protocol under which the validation studies were conducted.

Specimen selection and processing have been described previously. ${ }^{9,10}$ After microarray quality control using the Affymetrix Power Tools packages (Affymetrix, Santa Clara, CA), ${ }^{14}$ probe set summarization and normalization were performed with the SCAN algorithm, which normalizes each batch individually by modeling and removing probe- and array-specific background noise using only data from within each array. ${ }^{15}$

\section{Calculation of GC, Nomograms, and Combined Models}

The expression values for the 22 prespecified biomarkers that constitute the GC were extracted from the normalized data matrix and entered into the locked random forests algorithm with tuning and weighting parameters defined as reported previously. ${ }^{9,10,16}$ The GC read-out is a continuous risk score between 0 and 1 , with higher scores indicating a greater probability of metastasis. ${ }^{16} \mathrm{GC}$ scores were rounded to two significant digits.

Cancer of the Prostate Risk Assessment Postsurgical (CAPRA-S) score was indirectly derived from a regression equation using seven variables. ${ }^{17}$ Stephenson 5-year nomogram survival probability was calculated using eight clinicopathologic variables based on the locked Cox proportional hazards regression model. ${ }^{18}$ The combined GC plus CAPRA-S and GC plus Stephenson models were trained for predicting the metastasis end point and locked on an independent data set as reported previously. ${ }^{16}$ The training data set included 186 patients with 69 patients with metastatic disease. Overall, 47\%, $49 \%$, and $35 \%$ of patients had positive margins, extraprostatic extension, and seminal vesicle invasion, respectively.

\section{Statistical Analyses}

Age at RP, preoperative PSA level (log2 transformed), and time from RP to RT were considered continuous variables. Pathologic Gleason score $(\leq 7 v$ $>7$ ), extraprostatic extension (present $v$ absent), seminal vesicle invasion (present $v$ absent), surgical margin status (positive $v$ negative), and treatment modality (ART $v$ SRT) were considered categorical variables. In time-to-event analyses, event times were defined as the time from completion of RT to metastasis date.
The prognostic accuracy of the CAPRA-S, Stephenson nomogram, GC, and combined models was established according to time-dependent receiver operating characteristic curves for survival data using the nearest neighbor estimator described by Heagerty et al. ${ }^{19}$ Cumulative incidence curves were constructed using Fine-Gray competing risks analysis to estimate the risk of metastasis over time. ${ }^{20}$ As a result of the small number of events, penalized likelihood Cox regression methods (LASSO and Firth) were used for identification of the most prognostic risk factors to ensure the robustness of the analyses and avoid overestimation of the resulting hazard ratios (HRs). ${ }^{21,22}$ Decision curve analysis was used to determine the net benefit derived from the use of the GC, CAPRA-S, GC plus CAPRA-S model, and GC plus Stephenson model. ${ }^{23}$ The significance level was $P=.05$ for all statistical tests, and analyses were performed in R version 3.0 (http://www.r-project.org/).

\section{RESULTS}

The clinical characteristics of the study cohort are listed in Table 1. Seventy-two percent of men had extraprostatic extension, 35\% had seminal vesicle invasion, and $78 \%$ had positive margins. Twenty-one percent of patients had a Gleason score of $\geq 8$. Fifty-one percent of patients received ART ( $89 \%$ within 12 months of RP), and overall, patients received RT at a median of 5 months (range, 1 to 160 months)

Table 1. Demographics and Clinical Characteristics of Eligible Patients

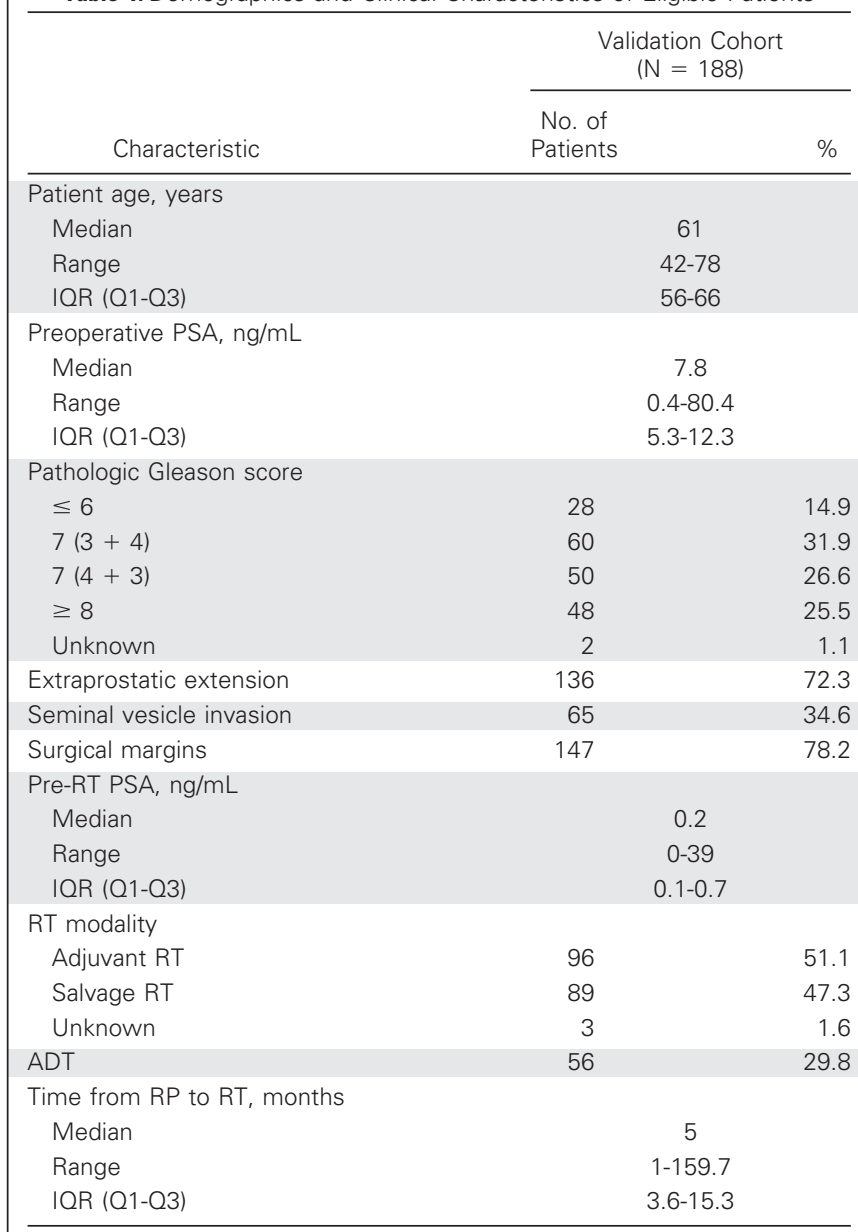

Abbreviations: ADT, androgen-deprivation therapy; IQR, interquartile range; PSA, prostate-specific antigen; RP, radical prostatectomy; RT, radiation therapy; Q, quartile. 
after RP. Thirty percent of patients received hormonal therapy with RT. The median follow-up times after RP and after RT were 10 and 8 years, respectively. Overall, 19 patients (10\%) developed metastasis after post-RP RT, with a median time to metastasis of 3 years (interquartile range, 1 to 5 years; Table 1 ).

Using the CAPRA-S scoring model, the majority of the patients were categorized as either at average risk (50\%) or high risk (45\%) for disease progression (Appendix Fig A1A, online only). In contrast, the rates of men with previously described cut points for low $(<0.4)$, average ( 0.4 to 0.6 ), and high ( $>0.6$ ) GC scores were $39 \%, 41 \%$, and $20 \%$, respectively (Appendix Fig A1B). GC scores had a modest correlation with Gleason score (Spearman's $\rho=0.26$; $P<.001$ ).

The survival concordance index (c-index) for predicting metastasis at 5 years after RT was 0.66 (95\% CI, 0.56 to 0.78 ) for the CAPRA-S model, 0.83 (95\% CI, 0.27 to 0.89 ) for the GC score, and 0.85 (95\% CI, 0.79 to 0.93 ) for the CAPRA-S plus GC model (Fig 1A). Similar results were observed using the Stephenson nomogram (Appendix Fig A2, online only). Of the 19 patients who developed metastasis, 16 patients $(84 \%)$ had average or high GC scores (GC $\geq 0.4)$, and two patients had the highest possible GC score (0.39) still categorized as low risk.

Consistent with the survival c-index, decision curve analysis showed that the models including GC (GC alone and GC plus CAPRA-S) were superior to clinicopathologic models (Fig 1B). Compared with scenarios where no prediction model would be used for a post-RP RT treatment decision (ie, treat all or treat none), the GCbased models had a higher net benefit than clinical models across a wide range of decision threshold probabilities (approximately $0 \%$ to $25 \%$ risk of metastasis). Furthermore, reclassification analysis shows that 71 patients (43\%) with average- and high-risk CAPRA-S scores had their risk downgraded to GC low risk, and notably, 68 (96\%) of these 71 patients remained metastasis free on study follow-up (Appendix Table A1, online only).

Univariable analysis demonstrated that GC, preoperative PSA levels, and RT modality were significant predictors of metastasis (Table 2). In multivariable analysis, only pre-RP PSA levels and GC were independent predictors of metastasis (Table 2). As a continuous variable, for every $10 \%$ increase in GC score, the HR for metastasis was 1.90 (95\% CI, 1.31 to $2.75 ; P<.001)$. When analyzed as a categorical variable, high GC scores $(>0.6)$ had an $\mathrm{HR}$ of $9.58(P=.013)$ compared with low GC scores $(<0.4)$ (Appendix Table A2, online only). Results of the multivariable analysis were confirmed using LASSO penalized regression for sparse data and rare events. Even with large values of the penalty parameter $\lambda$, GC had a nonzero hazard coefficient and was the first variable to enter the model, confirming its significance in predicting metastasis in multivariable analysis despite the few metastasis events in this cohort (Fig 1C). In a multivariable model that included GC and CAPRA-S, both of these variables were significant predictors of metastasis, with HRs of 1.69 (per 0.1-unit increase; $P<.001$ ) and 1.28 (per 1-unit increase; $P=.028$ ), respectively (Table 2).

Cumulative incidence plots depicted the estimated incidence of metastasis, after stratifying patients according to GC and CAPRA-S risk groups (Fig 2). The 5-year cumulative incidence rates of metastasis in patients with low, average, and high CAPRA-S scores were 13\%, $2 \%$, and $14 \%$, respectively $(P=.04)$. The 5 -year cumulative incidence rates of metastasis in patients with low, average, and high GC scores were $0 \%, 9 \%$, and $29 \%$, respectively $(P=.002)$.
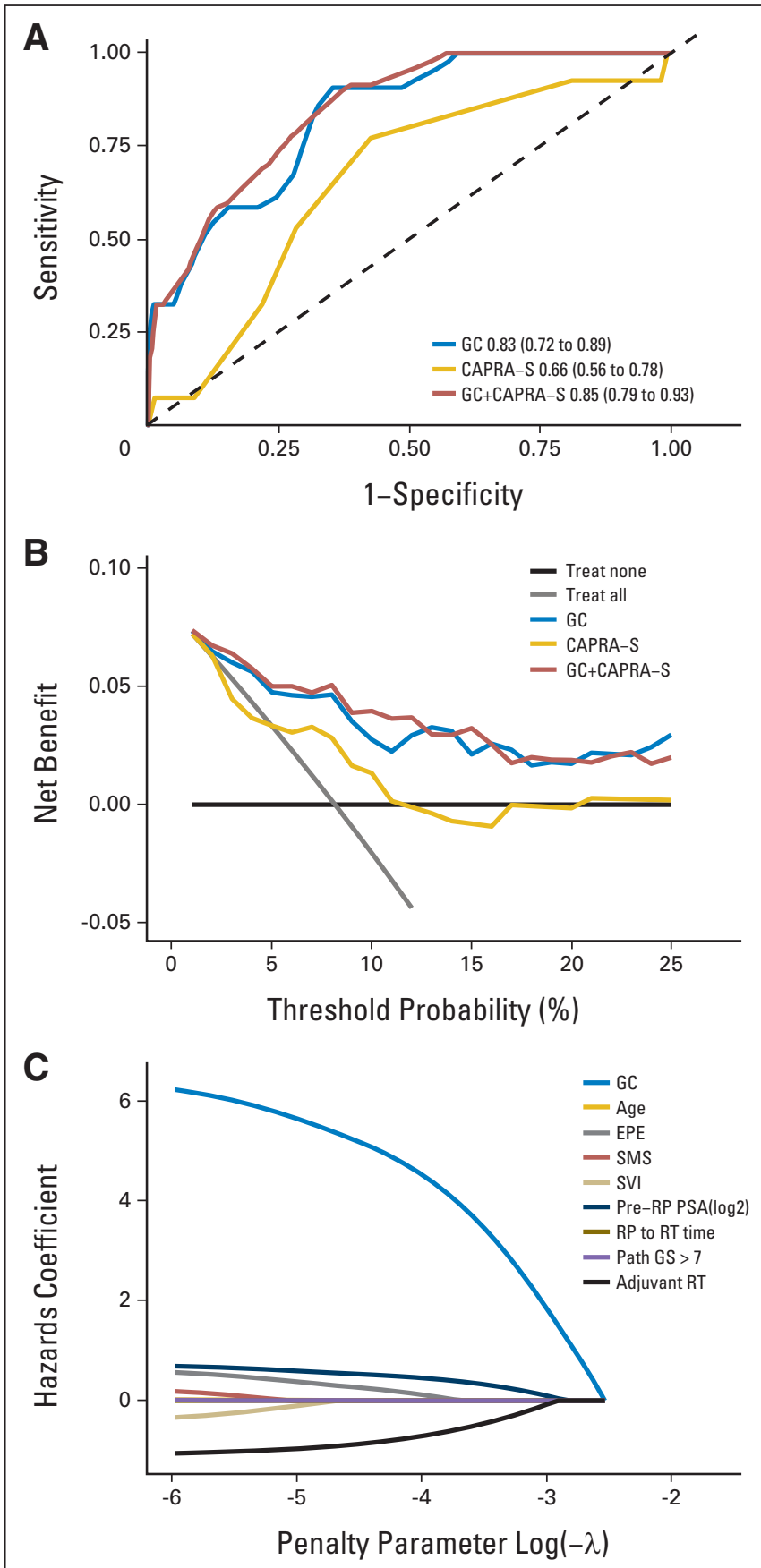

Fig 1. Discriminatory performance of the genomic classifier (GC) compared with clinical risk factors and Cancer of the Prostate Risk Assessment Postsurgical (CAPRA-S) score using different metrics. (A) GC has the highest survival concordance index compared with clinical models at 5 years after radiotherapy (RT). (B) Decision curve analysis for 5-year post-RT metastasis prediction shows that models including GC have the highest net benefit across $0 \%$ to $25 \%$ threshold probabilities. (C) Lasso hazards coefficient path of GC and clinical risk factors showing that GC is the first variable to enter the model as the penalty parameter is decreased. EPE, extraprostatic extension; GS, Gleason score; PSA, prostate-specific antigen; RP, radical prostatectomy; SMS, surgical margin status; SVI, seminal vesicle invasion.

Next, the prognostic models were evaluated for their ability to predict benefit from RT modality. Cumulative incidence plots for metastasis comparing ART with SRT were stratified by CAPRA-S and GC groups (Fig 3). The low and average CAPRA-S risk groups were 


\begin{tabular}{|c|c|c|c|c|c|c|}
\hline \multirow[b]{2}{*}{ Model and Variable } & \multicolumn{3}{|c|}{ UVA } & \multicolumn{3}{|c|}{ MVAt } \\
\hline & $\mathrm{HR}$ & $95 \% \mathrm{Cl}$ & $P$ & $\mathrm{HR}$ & $95 \% \mathrm{Cl}$ & $P$ \\
\hline \multicolumn{7}{|l|}{ Model I } \\
\hline Patient age, years & 1.02 & 0.96 to 1.09 & .5041 & 1.02 & 0.95 to 1.1 & .5643 \\
\hline Log2 preoperative PSA, ng/mL & 1.66 & 1.10 to 2.52 & .0158 & 2.12 & 1.31 to 3.45 & .0022 \\
\hline Pathologic Gleason score $\leq 7$ & Reference & & 1 & Reference & & 1 \\
\hline Pathologic Gleason score $>7$ & 2.36 & 0.89 to 6.00 & .0837 & 1.08 & 0.33 to 3.22 & .8889 \\
\hline Extraprostatic extension & 2.43 & 0.75 to 12.28 & .1489 & 1.67 & 0.45 to 9.05 & .4648 \\
\hline Seminal vesicle invasion & 1.46 & 0.55 to 3.71 & .4343 & 0.65 & 0.20 to 2.01 & .4561 \\
\hline Surgical margins & 0.64 & 0.23 to 2.10 & .424 & 1.31 & 0.43 to 4.62 & .6397 \\
\hline Time from RP to $R T$, months & 1.00 & 0.97 to 1.02 & .9802 & 1.01 & 0.97 to 1.04 & .7165 \\
\hline Adjuvant RT (reference: salvage RT) & 0.29 & 0.09 to 0.89 & .0219 & 0.37 & 0.11 to 1.05 & .0621 \\
\hline GC $\ddagger$ & 1.66 & 1.23 to 2.23 & $<.001$ & 1.90 & 1.31 to 2.75 & $<.001$ \\
\hline \multicolumn{7}{|l|}{ Model II } \\
\hline CAPRA-S§ & 1.31 & 1.05 to 1.63 & .0185 & 1.28 & 1.03 to 1.61 & .0282 \\
\hline $\mathrm{GC} \neq$ & 1.61 & 1.20 to 2.15 & $<.001$ & 1.69 & 1.24 to 2.31 & $<.001$ \\
\hline \multicolumn{7}{|c|}{$\begin{array}{l}\text { Abbreviations: CAPRA-S, Cancer of the Prostate Risk Assessment Postsurgical; GC, genomic classifier; HR, hazard ratio; MVA, multivariable analysis; PSA, } \\
\text { prostate-specific antigen; RP, radical prostatectomy; RT, radiation therapy; UVA, univariable analysis. } \\
\text { "According to Firth's penalized likelihood method where Cls are obtained via profile penalized likelihood. } \\
\text { tIn MVA, all available covariates were used and no variable selection was performed. For variable selection, LASSO was performed as shown in Figure 1C. } \\
\text { fGC reported per } 0.1 \text {-unit increase. } \\
\text { §CAPRA-S reported per 1-unit increase. }\end{array}$} \\
\hline
\end{tabular}

collapsed into one group, and the average and high GC score groups were similarly collapsed into one group as a result of limitations in sample size for this subset analysis. When comparing patients treated with ART versus SRT, the 5-year incidence of metastasis was $0 \%$ versus $7 \%(P=.02)$, respectively, for patients with CAPRA-S less than 5 (Fig 3A) and 7\% versus $21 \%(P=.1)$, respectively, for patients with CAPRA-S $\geq 5$ (Fig 3B). Stratified by GC, no differences in outcomes were observed comparing ART and SRT for patients with a GC score less than 0.4 ( $0 \% v 0 \%$, respectively; $P=.7$; Fig $3 \mathrm{C})$. In contrast, the results in patients with a GC score $\geq 0.4$ were significant and favored ART, with 5-year metastasis incidence of $6 \%$ versus $23 \%$ for SRT $(P=.008$; Fig 3D).
A sensitivity analysis was performed considering different PSA level thresholds (ie, $<0.1,0.1$ to 0.5 , and $>0.5 \mathrm{ng} / \mathrm{mL}$ ) at RT initiation (Figs $4 \mathrm{~A}$ and $4 \mathrm{~B}$ ). This analysis again showed that for patients in the low-risk GC group $(<0.4)$, no significant differences in cumulative incidence of metastasis were observed regardless of PSA level at RT $(P=.47)$. In the high-risk GC group, for patients who received RT when PSA was less than $0.1 \mathrm{ng} / \mathrm{mL}$ (these patients by contemporary criteria would be considered to be true ART patients), the cumulative incidence of metastasis at 5 years was $0 \%$, which is significantly better than the incidence of patients who received RT when PSA was between 0.1 and $0.5 \mathrm{ng} / \mathrm{mL}$ (ie, early SRT) or who received RT when PSA was greater than $0.5 \mathrm{ng} / \mathrm{mL}$ (ie, late SRT), who had a $12 \%$ and $26 \%$

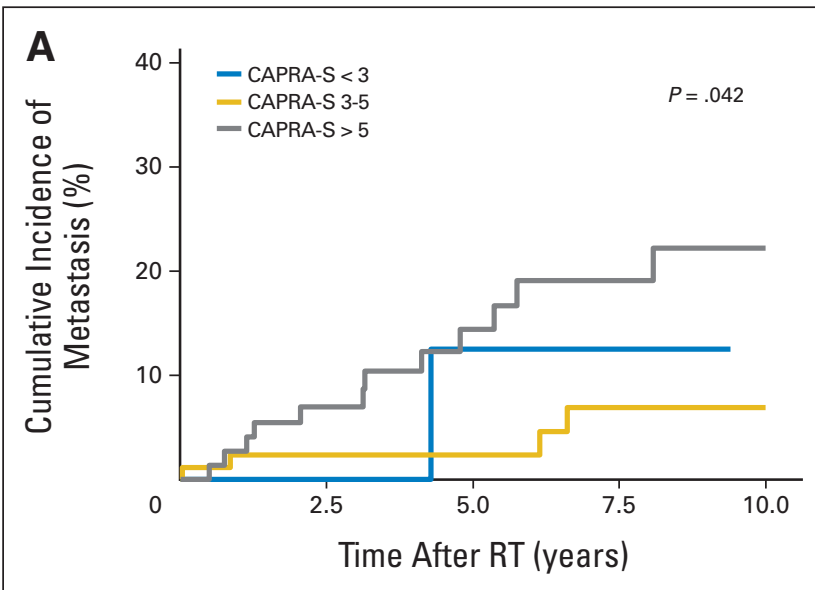

No. at risk

CAPRA-S $<3 \quad 10$

CAPRA-S $3-5 \quad 88$

CAPRA-S > 579

6
49
35

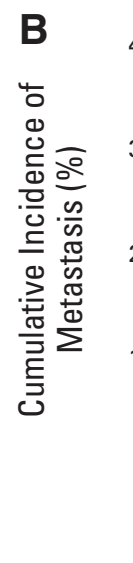

No. at risk

No. at risk
GC $<0.4$
GC $0.4-0.6$

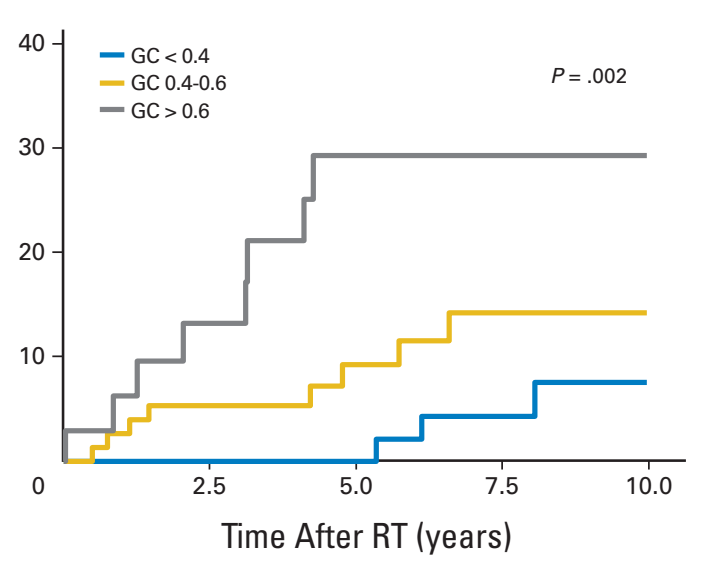

77
77
34
44
39
14 11 12

Fig 2. Cumulative incidence curves stratified by (A) Cancer of the Prostate Risk Assessment Postsurgical (CAPRA-S) score and (B) genomic classifier (GC) to evaluate their prognosis for postradiotherapy metastasis. RT, radiotherapy. 


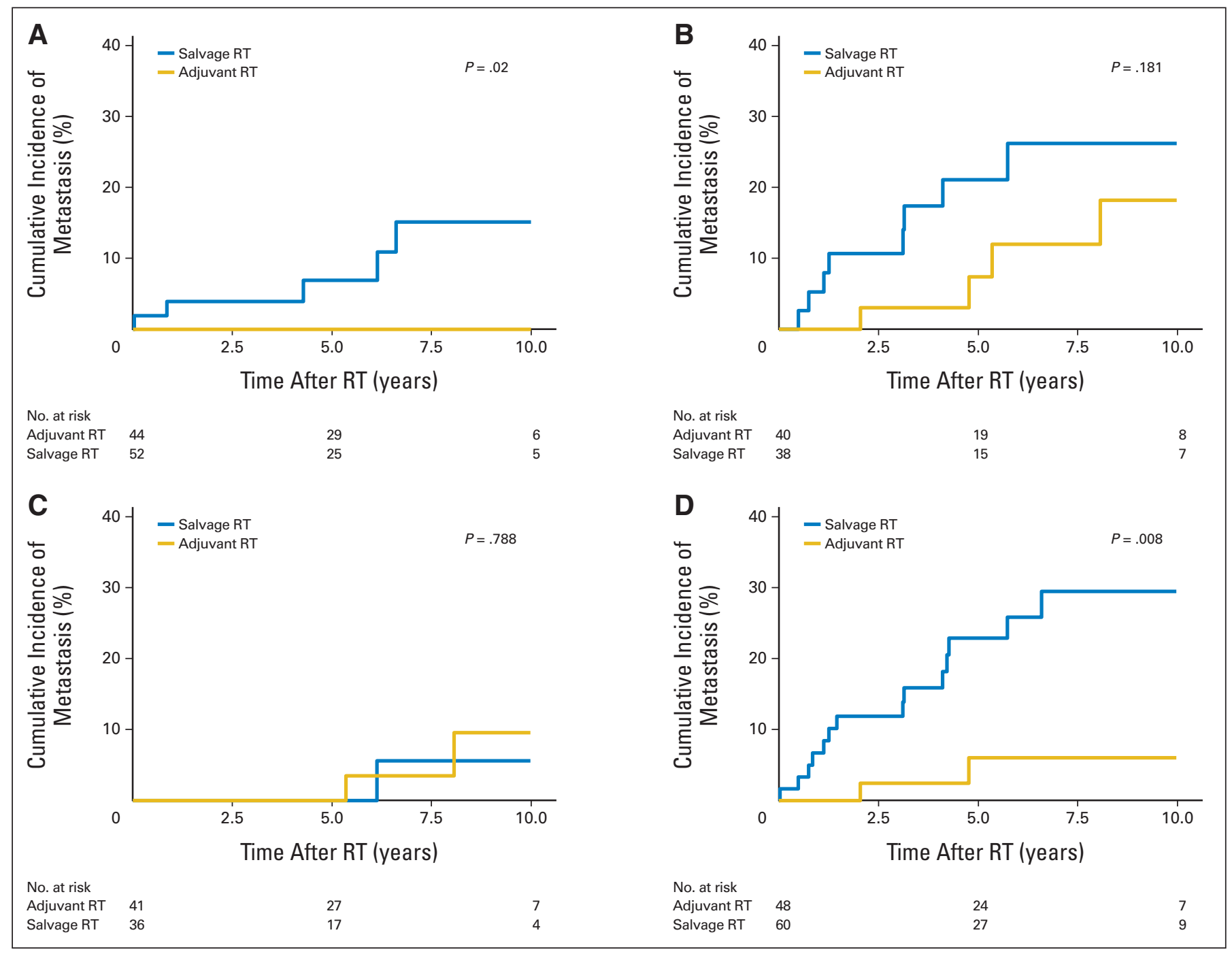

Fig 3. Cumulative incidence curves to evaluate benefit from adjuvant radiotherapy (RT) versus salvage RT stratified by (A and B) Cancer of the Prostate Risk Assessment Postsurgical (CAPRA-S) score and (C and D) genomic classifier (GC).

cumulative incidence of metastasis at 5 years after RT, respectively $(P=.02)$. Finally, Cox proportional hazards demonstrated that patients with higher GC scores who received ART had an $80 \%$ reduction in risk (HR, 0.20 ; $95 \% \mathrm{CI}, 0.04$ to $0.90 ; P<.04)$ compared with patients who received SRT (Appendix Table A3, online only). No benefit for ART was observed over SRT in patients with a low GC score (HR, $0.76 ; 95 \%$ CI, 0.11 to 5.76 ; $P<.8$ ).

\section{DISCUSSION}

Postprostatectomy RT significantly reduces the risks of PSA progression and local recurrence and may reduce the risk of distant metastases and PCa-specific mortality. ${ }^{24}$ However, there is a critical need within the genitourinary oncologic community to determine the optimal timing of postprostatectomy RT to avoid overtreatment and toxicities and realize the clinical benefits. Three prospective randomized trials comparing ART with initial observation for men with either pT3 disease or margin-positive (R1) resection (Southwest Oncology
Group 8794, ${ }^{25,26}$ European Organisation for Research and Treatment of Cancer 22911, ${ }^{27,28}$ and ARO 96-02/AUO AP/09/95 ${ }^{29,30}$ ) have demonstrated a benefit of ART in terms of bNED and local control at both 5- and 10-year follow-up. In addition, at 10 years, the Southwest Oncology Group trial demonstrated a benefit in overall survival and metastasis-free survival. ${ }^{26}$ However, this was not recapitulated in the European Organisation for Research and Treatment of Cancer trial, and the ARO 96-02 trial was not powered for overall survival. In these trials, the 5-year bNED rate for the observation arm was approximately $50 \%$, suggesting that adoption of ART for all men with positive-margin or $\mathrm{pT} 3$ disease would result in significant overtreatment. Furthermore, the use of ART has been shown to be associated with acute and late GI toxicity, urinary stricture, and incontinence, ${ }^{31}$ all representing potential patient management and quality-of-life outcomes challenges.

The current American Urologic Association/American Society for Radiation Oncology consensus guidelines reflect the challenge of counseling and decision making in this setting. ${ }^{32}$ Many 


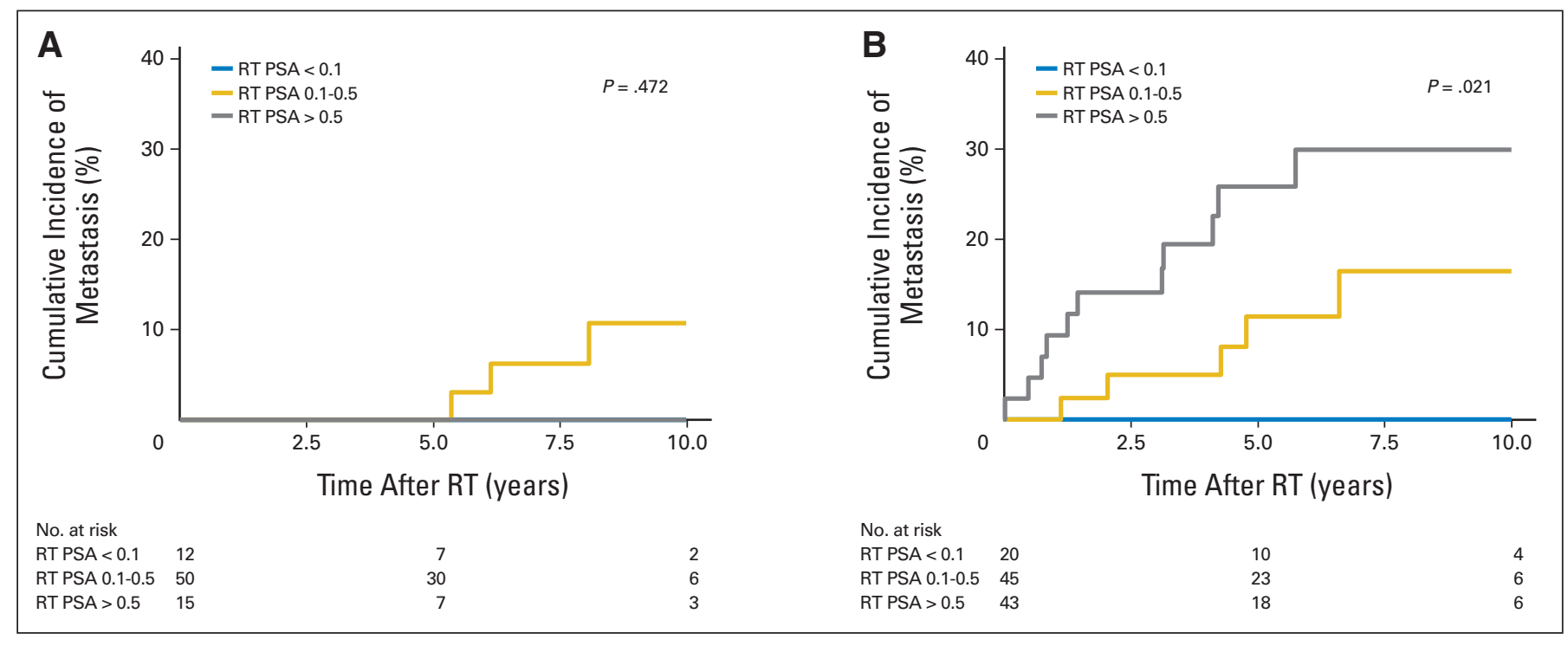

Fig 4. Cumulative incidence curves to evaluate benefit for three preradiotherapy prostate-specific antigen (PSA) levels $(<0.1,0.1$ to 0.5 , and $>0.5 \mathrm{ng} / \mathrm{mL})$ stratified by (A) low genomic classifier $(\mathrm{GC})$ score $(<0.4)$ and $(\mathrm{B})$ high $\mathrm{GC}$ score $(\geq 0.4)$. RT, radiotherapy.

experts have advocated for the identification of novel biomarkers to tailor treatment decisions. ${ }^{33,34}$ To that end, we used a multiinstitutional data set to examine the prognostic and predictive ability of a GC to determine the potential benefit of ART and SRT. We demonstrate that the GC is highly prognostic in the setting of postprostatectomy RT and that the GC may be a predictive marker that can help determine which patients will benefit from ART as opposed to SRT. This supports the importance of local therapy in the setting of presumed occult metastatic disease..$^{35,36}$

Within the literature, there are more than 100 published risk assessment tools, ${ }^{37}$ yet few are validated instruments. The most commonly referenced include the Stephenson postoperative nomogram ${ }^{18}$ and the CAPRA-S score. ${ }^{17}$ Recently, decision curve analysis has demonstrated that CAPRA-S score appropriately identified patients in whom adjuvant therapy is most appropriate, and CAPRA-S has been shown to be robust for prediction of PCa-specific mortality. ${ }^{38}$ In our study, first, we observed that GC downgraded risk in approximately $43 \%$ of CAPRA-S average- and high-risk patients to low risk GC, and $96 \%$ of these reclassified patients remained metastasis free on study follow-up. Accordingly, the c-index for predicting metastasis after RT was 0.66 for CAPRA-S but 0.83 for GC, with only a small gain to 0.85 for the combined model. Second, although we found that CAPRA-S retains significance in multivariable analysis with GC for predicting metastasis, it was observed that CAPRA-S score failed to discriminate patients who would benefit from ART. For patients with a less than $50 \%$ CAPRA-S risk of biochemical recurrence after $\mathrm{RP},{ }^{35} \mathrm{ART}$ was statistically associated with improved outcomes compared with SRT; however, for patients with a greater than 50\% risk of biochemical recurrence by the CAPRA-S model, no significant differences were observed. This is juxtaposed to the utilization of GC, in which there was no difference noted between patients treated with ART or SRT regardless of pre-RT PSA levels for patients with low GC scores, whereas there was a statistically significant decrease in development of metastases in men with high GC scores who received ART.

Currently, ART is being used in approximately $10 \%$ of patients with at least one adverse pathologic feature (positive-margin or pT3 disease) ${ }^{39-43}$ Given this low rate, some have questioned the extent to which preference-based and participatory decision making is occurring in routine clinical practice among patients and physicians. ${ }^{44}$ This study provides intriguing evidence to assist in the nuanced discussion of postprostatectomy treatment. This study adhered to the prospective collection of specimens before outcome ascertainment, ${ }^{45}$ and GC scores were determined with blinding to all clinical information.

There are a few limitations in this study. First, the data analyzed are retrospective, and the selection of ART as opposed to SRT varied among physicians and patients. Second, there were no concrete guidelines for the incorporation of androgen-deprivation therapy with postprostatectomy RT. Third, this study only included patients who received RT and thus could not identify a patient population in whom postprostatectomy RT could be withheld completely.

Despite these limitations, the findings of the study are particularly intriguing and provide a unique, more individualized approach in the management of postprostatectomy patients with adverse pathologic findings. Although a biomarker should not substitute for the shared patient-physician decision-making process, the integration of GC can provide additional insight into the aggressiveness of a man's $\mathrm{PCa}$ and more appropriately guide his postprostatectomy therapy selection. This study suggests that for men with a high GC score receiving SRT further intensification of therapy may be warranted; this is currently being examined in the Radiation Therapy Oncology Group 96-01 study, a prospective phase III randomized trial comparing SRT with SRT plus highdose bicalutamide. Given that this cohort consists of high-risk patients by clinicopathologic nomograms and the utilization of a GC allowed for significant downstaging, this study has major ramifications in terms of both potential for overtreatment and substantial cost savings to the US health care system. ${ }^{46}$ Thus, the GC is a valuable tool to aid in management of men with PCa undergoing prostatectomy. 


\section{AUTHORS' DISCLOSURES OF POTENTIAL CONFLICTS} OF INTEREST

Disclosures provided by the authors are available with this article at www.jco.org.

\section{AUTHOR CONTRIBUTIONS}

Conception and design: Robert B. Den, Kasra Yousefi, Edouard J. Trabulsi, Firas Abdollah, Adam P. Dicker, Costas D. Lallas, Elai Davicioni
Financial support: Elai Davicioni

Administrative support: Adam P. Dicker, Elai Davicioni

Provision of study materials or patients: Robert B. Den, Costas D. Lallas, Elai Davicioni

Collection and assembly of data: Robert B. Den, Kasra Yousefi, Elai Davicioni, R. Jeffrey Karnes

Data analysis and interpretation: Robert B. Den, Kasra Yousefi, Edouard J. Trabulsi, Firas Abdollah, Voleak Choeurng, Felix Y. Feng, Adam P. Dicker, Leonard G. Gomella, Elai Davicioni

Manuscript writing: All authors

Final approval of manuscript: All authors

\section{REFERENCES}

1. Silberstein $\mathrm{JL}$, Vickers $A J$, Power $N E$, et al: Reverse stage shift at a tertiary care center: Escalating risk in men undergoing radical prostatectomy. Cancer 117:4855-4860, 2011

2. Cary $K C$, Paciorek A, Fuldeore MJ, et al: Temporal trends and predictors of salvage cancer treatment after failure following radical prostatectomy or radiation therapy: An analysis from the CaPSURE registry. Cancer 120:507-512, 2014

3. Ost P, De Troyer B, Fonteyne V, et al: A matched control analysis of adjuvant and salvage high-dose postoperative intensity-modulated radiotherapy for prostate cancer. Int J Radiat Oncol Biol Phys 80:1316-1322, 2011

4. Trabulsi EJ, Valicenti RK, Hanlon AL, et al: A multi-institutional matched-control analysis of adjuvant and salvage postoperative radiation therapy for pT3-4NO prostate cancer. Urology 72:1298-1302. 2008

5. Briganti $A$, Wiegel $T$, Joniau $S$, et al: Early salvage radiation therapy does not compromise cancer control in patients with PT3NO prostate cancer after radical prostatectomy: Results of a matchcontrolled multi-institutional analysis. Eur Urol 62: 472-487, 2012

6. Parker C, Clarke N, Logue J, et al: RADICALS (Radiotherapy and Androgen Deprivation in Combination after Local Surgery). Clin Oncol (R Coll Radiol) 19:167-171, 2007

7. Richaud $P$, Sargos $P$, Henriques de Figueiredo $B$, et al: Postoperative radiotherapy of prostate cancer. Cancer Radiother 14:500-503, 2010

8. Pearse M, Fraser-Browne C, Davis ID, et al: A phase III trial to investigate the timing of radiotherapy for prostate cancer with high-risk features: Background and rationale of the RadiotherapyAdjuvant Versus Early Salvage (RAVES) trial. BJU Int 113:7-12, 2014 (suppl)

9. Den RB, Feng FY, Showalter TN, et al: Genomic prostate cancer classifier predicts biochemical failure and metastases in patients after postoperative radiation therapy. Int J Radiat Oncol Biol Phys 89:1038-1046, 2014

10. Karnes RJ, Bergstralh EJ, Davicioni E, et al: Validation of a genomic classifier that predicts metastasis following radical prostatectomy in an at risk patient population. J Urol 190:2047-2053, 2013

11. Mishra MV, Scher ED, Andrel J, et al: Adjuvant versus salvage radiation therapy for prostate cancer patients with adverse pathologic features: comparative analysis of long-term outcomes. Am J Clin Oncol [epub ahead of print on March 26, 2013]

12. Briganti A, Karnes RJ, Joniau S, et al: Prediction of outcome following early salvage radiotherapy among patients with biochemical recurrence after radical prostatectomy. Eur Urol 66:479-486, 2014
13. McShane $L M$, Altman $D G$, Sauerbrei $W$, et al: REporting recommendations for tumour MARKer prognostic studies (REMARK). Br J Cancer 100:229-235, 2006

14. Lockstone HE: Exon array data analysis using Affymetrix power tools and R statistical software. Brief Bioinform 12:634-644, 2011

15. Piccolo SR, Sun Y, Campbell JD, et al: A single-sample microarray normalization method to facilitate personalized-medicine workflows. Genomics 100:337-344, 2012

16. Erho N, Crisan A, Vergara IA, et al: Discovery and validation of a prostate cancer genomic classifier that predicts early metastasis following radical prostatectomy. PLoS One 8:e66855, 2013

17. Cooperberg MR, Hilton JF, Carroll PR: The CAPRA-S score: A straightforward tool for improved prediction of outcomes after radical prostatectomy. Cancer 117:5039-5046, 2011

18. Stephenson AJ, Scardino PT, Eastham JA, et al: Postoperative nomogram predicting the 10-year probability of prostate cancer recurrence after radical prostatectomy. J Clin Oncol 23:7005-7012, 2005

19. Heagerty PJ, Lumley T, Pepe MS: Timedependent ROC curves for censored survival data and a diagnostic marker. Biometrics 56:337-344, 2000

20. Fine JP, Gray RJ: A proportional hazards model for the subdistribution of a competing risk. J Am Stat Assoc 94:496-509, 1999

21. Tibshirani R: The lasso method for variable selection in the Cox model. Stat Med 16:385-395, 1997

22. Firth D: Bias reduction of maximum likelihood estimates. Biometrika 80:27, 1993

23. Vickers AJ, Elkin EB: Decision curve analysis: A novel method for evaluating prediction models. Med Decis Making 26:565-574, 2006

24. Swanson GP, Hussey MA, Tangen CM, et al: Predominant treatment failure in postprostatectomy patients is local: Analysis of patterns of treatment failure in SWOG 8794. J Clin Oncol 25:2225-2229, 2007

25. Thompson IM Jr, Tangen CM, Paradelo J, et al: Adjuvant radiotherapy for pathologically advanced prostate cancer: A randomized clinical trial. JAMA 296:2329-2335, 2006

26. Thompson IM, Tangen $\mathrm{CM}$, Paradelo J, et al: Adjuvant radiotherapy for pathological T3NOMO prostate cancer significantly reduces risk of metastases and improves survival: Long-term followup of a randomized clinical trial. J Urol 181:956-962, 2009

27. Bolla $M$, van Poppel $H$, Collette $L$, et al: Postoperative radiotherapy after radical prostatectomy: A randomised controlled trial (EORTC trial 22911). Lancet 366:572-578, 2005

28. Bolla M, van Poppel H, Tombal B, et al: Postoperative radiotherapy after radical prostatectomy for high-risk prostate cancer: Long-term results of a randomised controlled trial (EORTC trial 22911). Lancet 380:2018-2027, 2012
29. Wiegel $T$, Bottke $D$, Steiner $U$, et al: Phase III postoperative adjuvant radiotherapy after radical prostatectomy compared with radical prostatectomy alone in PT3 prostate cancer with postoperative undetectable prostate-specific antigen: ARO 96-02/AUO AP 09/95. J Clin Oncol 27:2924-2930, 2009

30. Wiegel T, Bartkowiak D, Bottke D, et al: Adjuvant radiotherapy versus wait-and-see after radical prostatectomy: 10-year follow-up of the ARO 96-02/AUO AP 09/95 trial. Eur Urol 66:243-250, 2014

31. Daly $T$, Hickey BE, Lehman $M$, et al: Adjuvant radiotherapy following radical prostatectomy for prostate cancer. Cochrane Database Syst Rev 12: CD007234, 2011

32. Thompson IM, Valicenti RK, Albertsen $P$, et al: Adjuvant and salvage radiotherapy after prostatectomy: AUA/ASTRO guideline. J Urol 190:441-449, 2013

33. Stephenson AJ, Bolla M, Briganti $A$, et al: Postoperative radiation therapy for pathologically advanced prostate cancer after radical prostatectomy. Eur Urol 61:443-451, 2012

34. Thompson IM, Tangen CM, Klein EA: Is there a standard of care for pathologic stage T3 prostate cancer? J Clin Oncol 27:2898-2899, 2009

35. Widmark A, Klepp O, Solberg A, et al: Endocrine treatment, with or without radiotherapy, in locally advanced prostate cancer (SPCG-7/SFUO-3): An open randomised phase III trial. Lancet 373:301-308, 2009

36. Warde $P$, Mason M, Ding K, et al: Combined androgen deprivation therapy and radiation therapy for locally advanced prostate cancer: A randomised, phase 3 trial. Lancet 378:2104-2111, 2011

37. Shariat SF, Kattan MW, Vickers AJ, et al: Critical review of prostate cancer predictive tools. Future Oncol 5:1555-1584, 2009

38. Punnen S, Freedland SJ, Presti JC Jr, et al: Multi-institutional validation of the CAPRA-S score to predict disease recurrence and mortality after radical prostatectomy. Eur Urol 65:1171-1177, 2014

39. Sheets NC, Hendrix LH, Allen IM, et al: Trends in the use of postprostatectomy therapies for patients with prostate cancer: A Surveillance, Epidemiology, and End Results Medicare analysis. Cancer 119:3295-3301, 2013

40. Schreiber D, Rineer J, Yu JB, et al: Analysis of pathologic extent of disease for clinically localized prostate cancer after radical prostatectomy and subsequent use of adjuvant radiation in a national cohort. Cancer 116:5757-5766, 2010

41. Kalbasi A, Swisher-McClure S, Mitra N, et al: Low rates of adjuvant radiation in patients with nonmetastatic prostate cancer with high-risk pathologic features. Cancer 120:3089-3096, 2014

42. Ghia AJ, Shrieve DC, Tward JD: Adjuvant radiotherapy use and patterns of care analysis for margin-positive prostate adenocarcinoma with extracapsular extension: Postprostatectomy adjuvant radiotherapy—A SEER analysis. Urology 76:11691174,2010 
43. Hoffman $\mathrm{KE}$, Nguyen $\mathrm{PL}$, Chen $\mathrm{MH}$, et al: Recommendations for post-prostatectomy radiation therapy in the United States before and after the presentation of randomized trials. J Urol 185:116120, 2011
44. Abernethy AP, Etheredge LM, Ganz PA, et al: Rapid-learning system for cancer care. J Clin Oncol 28:4268-4274, 2010

45. Pepe MS, Feng $Z$, Janes $H$, et al: Pivotal evaluation of the accuracy of a biomarker used for classification or prediction: Standards for study design. J Natl Cancer Inst 100:1432-1438, 2008

46. Wilson LS, Tesoro R, Elkin EP, et al: Cumulative cost pattern comparison of prostate cancer treatments. Cancer 109:518-527, 2007

\section{GLOSSARY TERMS}

Cancer of the Prostate Risk Assessment (CAPRA) score: a 0 to 10 score based on a multivariable Cox model that predicts biochemical and clinical (metastasis and mortality) end points after primary treatment for prostate cancer. A postsurgical version (CAPRA-S) offers improved prediction of the same end points after radical prostatectomy.

Cox proportional hazards regression model: a statistical model for regression analysis of censored survival data, examining the relationship of censored survival distribution to one or more covariates. This model produces a baseline survival curve, covariate coefficient estimates with their standard errors, risk ratios, 95\% CIs, and significance levels.

decision curve analysis: an approach to evaluating the discrimination and calibration of different prognostic tests or models. A decision curve plots net benefit for a given model across a range of threshold probabilities. Net benefit is calculated as true positives minus false positives, with the false-positive term weighted by the threshold probability. The threshold probability indicates the likelihood of a positive finding at which an intervention would be undertaken, given the results of the test or model.

prostate-specific antigen (PSA): a protein produced by cells of the prostate gland. The blood level of prostate-specific antigen (PSA) is used as a tumor marker for men who may be suspected of having prostate cancer. Most physicians consider 0 to $4.0 \mathrm{ng} / \mathrm{mL}$ to be the normal range. Levels of 4 to 10 and 10 to $20 \mathrm{ng} / \mathrm{mL}$ are considered slightly and moderately elevated, respectively. PSA levels have to be complemented with other tests to make a firm diagnosis of prostate cancer.

REMARK criteria: guidelines for reporting tumor marker studies, which include a statement of objectives and a description of patient population and treatments received, biologic materials, and assay methods. Criteria also include guidelines for reporting data, results, and discussion. 


\section{AUTHORS' DISCLOSURES OF POTENTIAL CONFLICTS OF INTEREST}

Genomic Classifier Identifies Men With Adverse Pathology After Radical Prostatectomy Who Benefit From Adjuvant Radiation Therapy

The following represents disclosure information provided by authors of this manuscript. All relationships are considered compensated. Relationships are self-held unless noted. I = Immediate Family Member, Inst = My Institution. Relationships may not relate to the subject matter of this manuscript. For more information about ASCO's conflict of interest policy, please refer to www.asco.org/rwc or jco.ascopubs.org/site/ifc.

\section{Robert B. Den}

Research Funding: GenomeDx Biosciences

Travel, Accommodations, Expenses: GenomeDx Biosciences

\section{Kasra Yousefi}

Employment: GenomeDx Biosciences

Stock or Other Ownership: GenomeDx Biosciences

Edouard J. Trabulsi

Consulting or Advisory Role: GenomeDx Biosciences, Janssen, Medivation, CE Outcomes, Photocure, Dendreon

Firas Abdollah

Consulting or Advisory Role: GenomeDx Biosciences

\section{Voleak Choeurng}

Employment: GenomeDx Biosciences

Stock or Other Ownership: GenomeDx Biosciences

\section{Felix Y. Feng}

Consulting or Advisory Role: Medivation, Astellas, GenomeDx Biosciences, Celgene

Research Funding: Varian, Celgene, Medivation/Astellas

\section{Adam P. Dicker}

Consulting or Advisory Role: Merck KGaA, Vertex, Merck, Glenview Consulting, GenomeDx Biosciences
Speakers' Bureau: Bayer

Travel, Accommodations, Expenses: Bayer, Varian Medical Systems, Merck KGaA

Other Relationship: NRG Oncology

\section{Costas D. Lallas}

No relationship to disclose

Leonard G. Gomella

Consulting or Advisory Role: Astellas Pharma, Janssen Pharmaceuticals, Algeta/Bayer

Research Funding: Astellas Pharma (Inst), Janssen Pharmaceuticals (Inst), Myriad Genetics (Inst)

Patents, Royalties, Other Intellectual Property: Prostate cancer biomarker (Inst)

Elai Davicioni

Employment: GenomeDx Biosciences

Leadership: GenomeDx Biosciences

Stock or Other Ownership: GenomeDx Biosciences

R. Jeffrey Karnes

Travel, Accommodations, Expenses: GenomeDx Biosciences 


\section{Acknowledgment}

We thank Darby J.S. Thompson, PhD (EMMES Canada), Christine Buerki, PhD, Heesun Shin, PhD, Jason Alter, PhD, Mercedeh Ghadessi, and Penelope Wood of GenomeDx Biosciences for useful comments and advice relating to study design and analysis. We also thank Rachel Carlson (Mayo Clinic) for her assistance in data preparation.

\section{Appendix}

\begin{tabular}{|c|c|c|c|c|c|}
\hline \multirow[b]{2}{*}{ Model } & \multicolumn{3}{|c|}{ CAPRA-S Score (No. of patients) } & \multicolumn{2}{|c|}{ Total Patients } \\
\hline & $<3$ & $3-5$ & $>5$ & No. & $\%$ \\
\hline \multicolumn{6}{|l|}{$\overline{\text { GC score }}$} \\
\hline$<0.4$ & 2 & 42 & 29 & 73 & 41.2 \\
\hline Metastasis & 0 & 1 & 2 & & \\
\hline $0.4-0.6$ & 5 & 31 & 35 & 71 & 40.1 \\
\hline Metastasis & 0 & 1 & 5 & & \\
\hline$>0.6$ & 3 & 15 & 15 & 33 & 18.6 \\
\hline Metastasis & 1 & 2 & 5 & & \\
\hline \multicolumn{6}{|l|}{ Total patients } \\
\hline No. & 10 & 88 & 79 & $177^{*}$ & \\
\hline$\%$ & 5.6 & 49.7 & 44.6 & & \\
\hline
\end{tabular}

Table A2. Results of MVA Cox* Proportional Hazards Analysis of GC (categorical, $<0.4,0.4-0.6$, and $>0.6$ ) and Clinical Risk Factors

\begin{tabular}{|c|c|c|c|}
\hline Variable & HR & $95 \% \mathrm{Cl}$ & $P$ \\
\hline Log2 preoperative PSA, ng/mL & 2.04 & 1.25 to 3.39 & .0046 \\
\hline Pathologic Gleason score $\leq 7$ & Reference & & 1 \\
\hline Pathologic Gleason score $>7$ & 1.67 & 0.54 to 4.98 & .3621 \\
\hline Extraprostatic extension & 1.54 & 0.41 to 8.32 & .5464 \\
\hline Seminal vesicle invasion & 0.61 & 0.18 to 1.96 & .4139 \\
\hline Surgical margins & 1.21 & 0.39 to 4.32 & .7495 \\
\hline \multicolumn{4}{|l|}{ GC score } \\
\hline$<0.4$ & Reference & & 1 \\
\hline $0.4-0.6$ & 2.29 & 0.55 to 11.11 & .258 \\
\hline$>0.6$ & 9.58 & 2.38 to 47.02 & .0013 \\
\hline
\end{tabular}

Abbreviations: GC, genomic classifier; HR, hazard ratio; MVA, multivariable analysis; PSA, prostate-specific antigen; RP, radical prostatectomy, RT, radiation therapy. *Using Firth's penalized likelihood method.

\begin{tabular}{|c|c|c|c|}
\hline \multicolumn{4}{|l|}{ Model I (GC $<0.4$ subset) } \\
\hline Adjuvant RT & 0.76 & 0.11 to 5.46 & .787 \\
\hline \multicolumn{4}{|l|}{ Model II (GC $\geq 0.4$ subset) } \\
\hline Salvage RT & Reference & & 1 \\
\hline
\end{tabular}




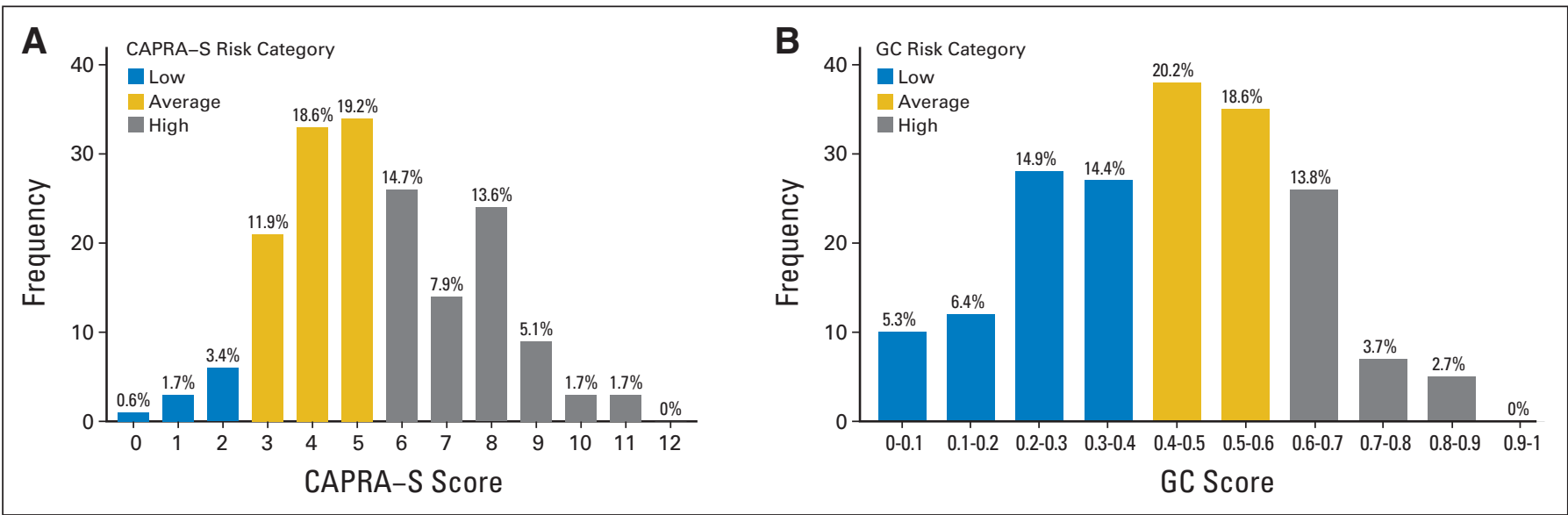

Fig A1. Risk score distribution of study patients based on (A) Cancer of the Prostate Risk Assessment Postsurgical (CAPRA-S) score and (B) genomic classifier (GC).

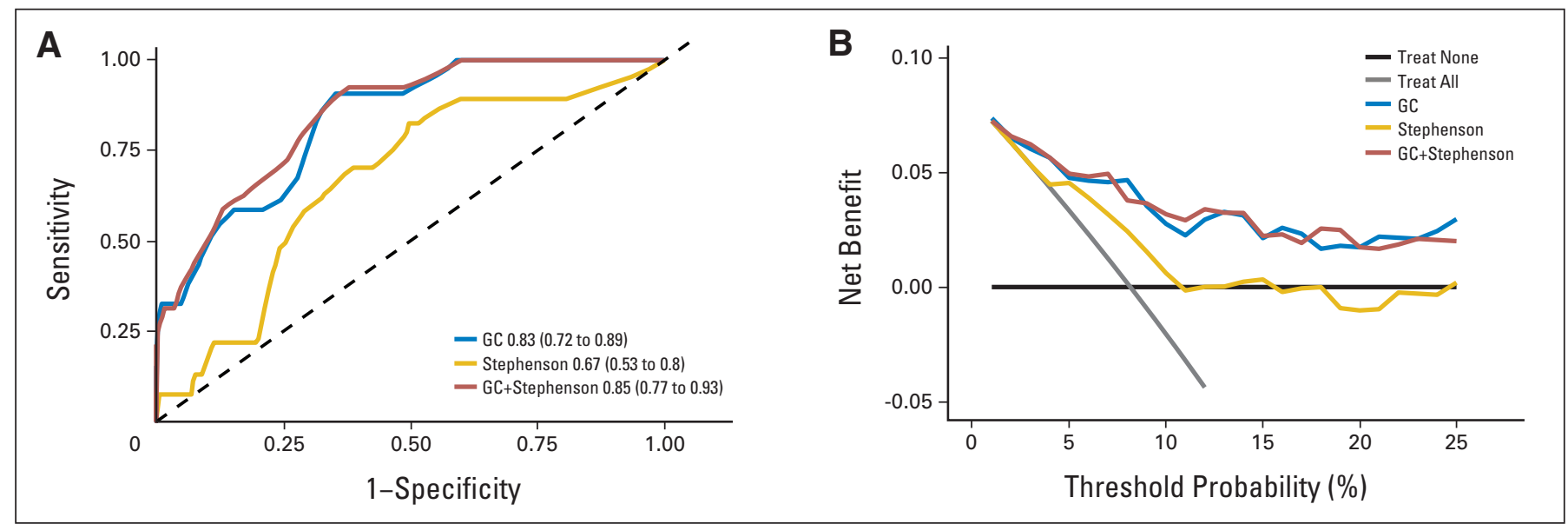

Fig A2. Discriminatory performance of genomic classifier (GC) compared with Stephenson nomogram. (A) GC has the highest survival concordance index compared with clinical models at 5 years after radiotherapy (RT). (B) Decision curve analysis for 5-year post-RT metastasis prediction shows that models including GC have the highest net benefit across $0 \%$ to $25 \%$ threshold probabilities. 